\title{
Using multiple chains in cross-correlation receivers to improve sensitivity
}

\author{
M. R. M. Mikhael, M. S. Oude Alink and A. B. J. Kokkeler \\ University of Twente, The Netherlands
}

\begin{abstract}
Cross-correlation can be used in energy detection applications, such as spectrum analyzers, but also frequency shift keying (FSK) receivers, to improve noise suppression. To achieve higher signal-to-noise ratio (SNR), integration in time may be used, but could make it rather slow for communication purposes. In order to achieve better data-rates in low SNR conditions, we propose to use multiple chains instead of the traditional two chains. In this paper, we show an analysis of the SNR improvement and the power consumption penalty for BFSK modulation when using more chains. It shows that for low noise correlation between the chains, the improvement in sensitivity is proportional to the number of chains. Also, we develop a figureof-merit to evaluate the optimum number of chains for different parameters of the receiver design. Furthermore, two examples from literature are analyzed. At their optimum number of chains, they both show $\sim 6 \mathrm{~dB}$ improvement in sensitivity with similar or even better figure-of-merit.
\end{abstract}

Index Terms-Cross-correlation, noise suppression, energy detection, FSK modulation, figure-of-merit.

\section{INTRODUCTION}

Energy detection receivers are used in many wireless applications such as spectrum analyzers, frequency shift keying (FSK) receivers and cognitive radios which use energy detection for spectrum sensing. Improving sensitivity and energy efficiency in energy detection receivers would improve the performance and reduce energy consumption of the whole system. In these applications, the noise of the receiver degrades the performance of the detector and reduces the detection probability or increases the bit-error rate (BER). In [1], the trade-off between sensitivity and power consumption of the receiver is discussed where in order to reduce the noise contribution of the receiver, its power consumption needs to be increased.

In [2], cross-correlation (XC) is proposed for energy detection for integrated spectrum analyzers. It is realized by using two different RF chains in the receiver and correlating their outputs to reduce the output noise assuming low correlation between the noise contributions of the two individual chains. This technique was shown to be more energy-efficient than using auto-correlation (AC) with one RF chain at low signalto-noise ratio (SNR) for the same performance. In [3], XC is proposed for non-coherent FSK receivers which shows about 2dB SNR improvement compared to traditional architectures but with almost twice the energy consumption per received bit.

In order to improve sensitivity and achieve the required BER in low SNR scenarios, integration in time may be needed by transmitting the same symbol several times which, in most

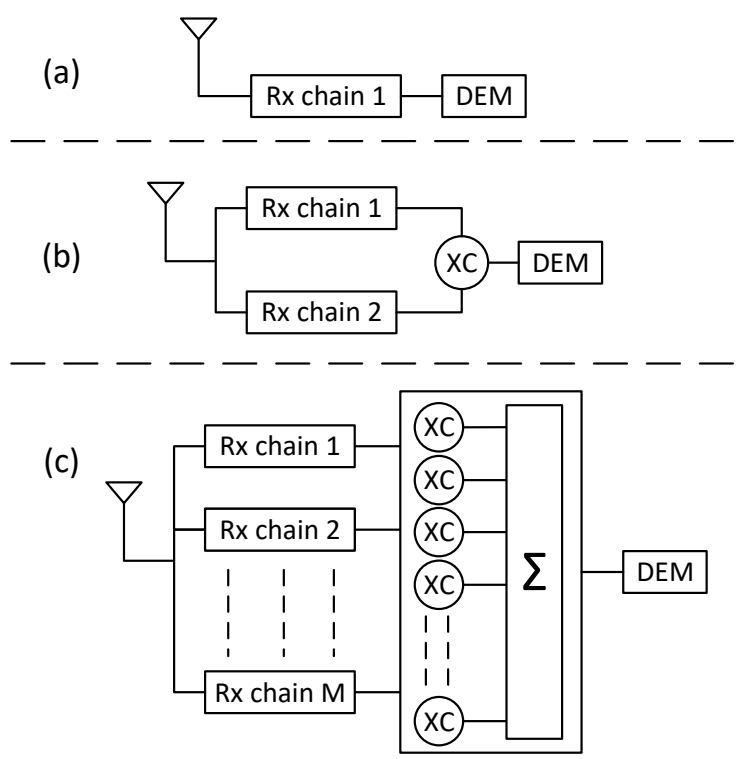

Fig. 1. (a) Traditional architecture of an energy detection FSK receiver using one RF chain, (b) Using XC with two chains, (c) Proposed architecture: increasing the number of chains and correlators for the $\mathrm{XC}$.

cases, has low energy efficiency. For double the time, the noise suppression is improved by about $1.5 \mathrm{~dB}$ using XC [4], while the energy spent per bit will be doubled $(+3 \mathrm{~dB})$. This makes integration in time rather slow, energy-inefficient and unsuitable for many applications that require higher data-rates.

In this paper, we propose to use more chains in parallel which allows using more correlators simultaneously, as shown in Fig. 1. The summation of the correlators output can then be fed to the demodulator (DEM) for the FSK signal demodulation. Sensitivity is then increased at the expense of extra hardware rather than extra integration time. This will allow this technique to be used in higher data-rate applications and becomes, in particular cases as will be shown later, more energy-efficient. By dynamically controlling which parts of the hardware are powered up, a direct trade-off between the receiver power consumption and its sensitivity is made possible.

In section II, the proposed architecture is discussed. In order to prove the merit of this implementation, the SNR at the input of the receiver required to achieve a specific BER is analyzed in section III. Also, the power consumption of the extra hardware is estimated and a figure-of-merit (FoM) is 
developed to determine whether using $\mathrm{XC}$ with multiple chains would be energy efficient. In section IV, Matlab simulations are performed to validate the analysis results. Furthermore, two examples from literature are analyzed in case of using more chains and its effect on the SNR, showing the practical applicability of this idea.

\section{SYSTEM DESCRIPTION}

Fig. 2 shows the proposed implementation of the FSK receiver in case of using five chains. In this implementation, $\mathrm{XC}$ is applied pairwise between the individual outputs of each chain in the frequency domain (FXC implementation). This can be achieved by taking an FFT of each chain output and for each bin, multiplying one of their outputs with the corresponding complex conjugate of the other. As shown, ten different unique XCs can be performed in this case. In general, $M$ chains would allow up to $M(M-1) / 2$ different XCs, i.e. the number of available XCs increases at a faster rate than the number of chains. The corresponding bins of XCs can be summed to suppress the noise compared to any one $\mathrm{XC}$ alone. The AC is not used since its estimate is biased and results in a much more complicated analysis.

In the proposed implementation, we are using FSK modulation with sinusoidal signals at different frequencies to modulate the data. Ideally, it appears as a delta function in its designated bin of the FFT. Furthermore, we will assume Gaussian noise throughout this paper. After the FFT, the noise will still follow a Gaussian distribution. The SNR is defined as the signal power divided by the noise power in its FFT bin.

The output of the adder which sums the correlators results, is then fed to the FSK demodulator which compares the energy in the different frequencies to demodulate the signal [3]. Sharing one antenna is useful to prevent signal de-correlation and offsets between chains. Assuming identical chains, the signal will only reside in the real component of the output (due to multiplication with the complex conjugate), so the imaginary component can be discarded. Using one antenna would mean that the input impedance of each chain would depend not only on the antenna impedance but also on the number of chains. For 5 chains and a $50 \Omega$ antenna, for example, the input impedance of each chain would be designed as $250 \Omega$ to ensure impedance matching. Increasing the input impedance may result in a small increase in the noise figure of the chain but it may also result in improved linearity. In [2], using $100 \Omega$ chain, instead of the $50 \Omega$ chain, increases the noise figure by $\sim 0.8 \mathrm{~dB}$ and IIP3 by $\sim 0.5 \mathrm{~dB}$.

The main limitation in our system is the correlated noise between chains. In the worst case of having fully correlated noise, all the improvements of this implementation would be lost. With one antenna, the capability of XC to suppress noise is limited to only the noise produced in the individual chains themselves, not to the noise from the antenna as, like the signal, it is fully correlated between all the chains. For receivers with low noise figure (NF), a large portion of the noise in each chain is from the shared antenna and is fully correlated. This may limit the SNR improvement from

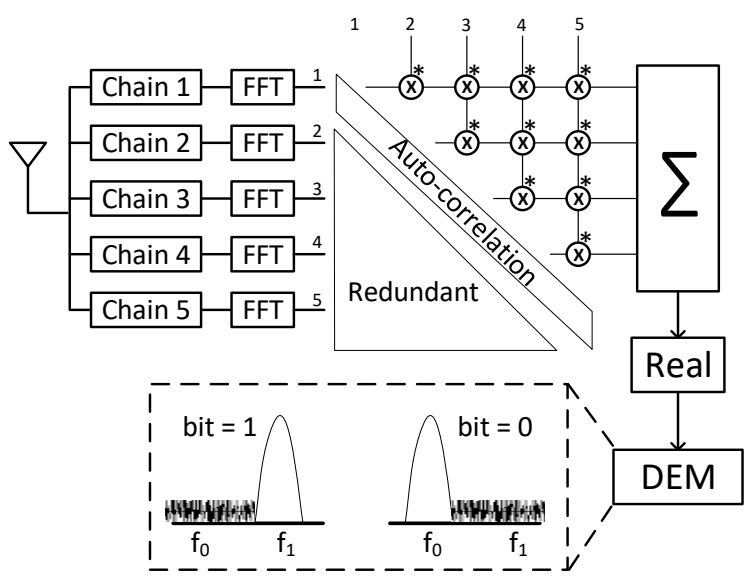

Fig. 2. System design of the proposed architecture for five chains. The FSK demodulator is fed with the energy of the spectrum and it demodulates the signal based on the highest energy in the used frequency bins.

increasing the number of chains. However, for designs with higher NF, thus potentially lower power consumption (and noise correlation), using multiple chains can be a very useful alternative to improve sensitivity.

In order for this system to be more energy-efficient, some components can be shared between chains with limited effect on the noise correlation. An example for this is the local oscillator (LO) which can be shared between different chains (Note that the LO would need to drive higher impedance in this case, which would increase its power consumption. Accounting for this increase, is discussed in section III).

\section{SySTEM MODEL AND ANALYSIS}

\section{A. Noise suppression}

Fig. 3 shows the mathematical model of the system described in section II. All the variables are in the frequency domain. The assumptions and notations used in the figure are as follows:

- $s$ is a variable representing the signal that is added to all the chains and its power is a constant $\sigma_{s}^{2}$;

- $n_{x}$ is a random variable representing circularly symmetric complex Gaussian (CSCG) noise added to all the chains where $n_{x} \sim \mathcal{C N}\left(0, \sigma_{\text {nx }}^{2}\right)$;

- $n_{c}(i)$ is a random variable representing CSCG noise added only to chain $i$ where $n_{c}(i) \sim \mathcal{C N}\left(0, \sigma_{\text {nc }}^{2}\right)$;

- The covariance $\operatorname{Cov}\left(n_{c}\left(i_{1}\right), n_{c}\left(i_{2}\right)\right)=0, \forall i_{1} \neq i_{2}$;

- $\operatorname{Cov}\left(n_{x}, n_{c}(i)\right)=0, \forall i$

- $n_{t}(i)$ represents the total noise in the chain $i: n_{t}(i)=$ $n_{x}+n_{c}(i)$. Thus, $n_{t}(i) \sim \mathcal{C N}\left(0, \sigma_{n}^{2}\right)$ where $\sigma_{n}^{2}=\sigma_{\mathrm{nx}}^{2}+$ $\sigma_{\mathrm{nc}}^{2}$.

The reason for using two different random variables $n_{x}$ and $n_{c}(i)$ to represent the noise in the chains is to control the noise correlation within the chains by the ratio of their variances. 


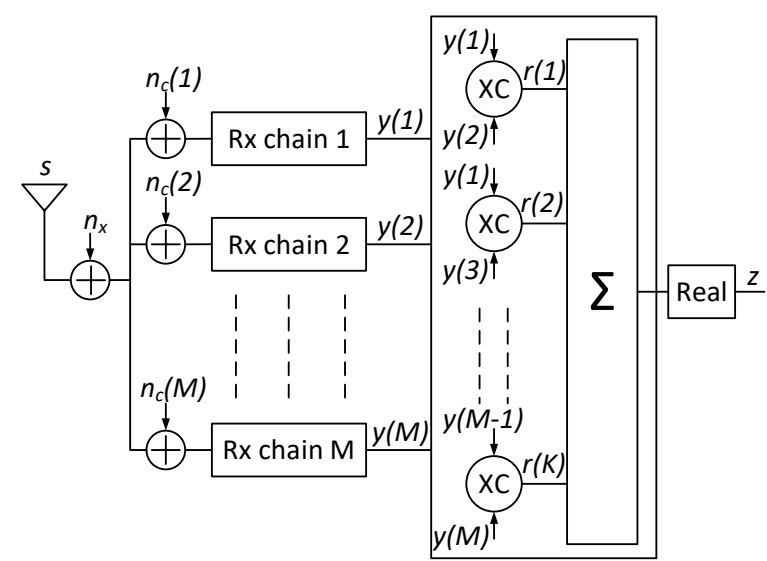

Fig. 3. Mathematical noise model for the proposed architecture using more chains assuming correlated noise between them.

To quantify the correlation, the correlation coefficient between the total noise in chains $i_{1}$ and $i_{2}$ for $i_{1} \neq i_{2}$ is

$$
\begin{aligned}
\rho_{i 1, i 2} \triangleq & \frac{\operatorname{Cov}\left(n_{t}\left(i_{1}\right), n_{t}\left(i_{2}\right)\right)}{\sqrt{\operatorname{Var}\left(n_{t}\left(i_{1}\right)\right) \operatorname{Var}\left(n_{t}\left(i_{2}\right)\right)}}= \\
& \frac{\mathrm{E}\left(\left(n_{x}+n_{c}\left(i_{1}\right)\right)\left(n_{x}+n_{c}\left(i_{2}\right)\right)^{*}\right)}{\sqrt{\left(\sigma_{n}^{2}\right)\left(\sigma_{n}^{2}\right)}}=\frac{\sigma_{\mathrm{nx}}^{2}}{\sigma_{n}^{2}}
\end{aligned}
$$

where $*$ denotes complex conjugation.

Since $\rho_{i 1, i 2}$ is identical for all pairs, we can write

$$
\begin{gathered}
\rho_{i 1, i 2}=\rho \quad \forall i_{1} \neq i_{2} \\
\sigma_{\mathrm{nx}}^{2}=\rho \sigma_{n}^{2}, \sigma_{\mathrm{nc}}^{2}=(1-\rho) \sigma_{n}^{2}
\end{gathered}
$$

Note that $\rho$ in our model represents the correlation between the noise in two different chains and does not take into account the signal. Due to the same correlated noise variable $n_{x}, 0 \leq$ $\rho \leq 1$ : $\rho$ cannot be negative in our model, nor can it have an imaginary component.

In this analysis, we are looking at the signal and noise after the FFT in the frequency domain. Without loss of generality, we will assume signal $s$ is real (i.e. its phase is defined as zero). The noise variables are defined as:

$$
\begin{gathered}
n_{x}=x_{r}+\mathrm{j} x_{m} \\
n_{c}(i)=c_{r}(i)+\mathrm{j} c_{m}(i)
\end{gathered}
$$

where both $x_{r}$ and $x_{m} \sim \mathcal{N}\left(0, \sigma_{\mathrm{nx}}^{2} / 2\right)$, and $\operatorname{Cov}\left(x_{r}, x_{m}\right)=$ 0 . Similarly, both $c_{r}(i)$ and $c_{m}(i) \sim \mathcal{N}\left(0, \sigma_{\mathrm{nc}}^{2} / 2\right)$, and $\operatorname{Cov}\left(c_{r}(i), c_{m}(i)\right)=0$.

Using these definitions, the output of each chain $y(i)$, the output of each correlator $r(k)$ and the total sum $z$ are evaluated.

$$
\begin{gathered}
y(i)=s+n_{c}(i)+n_{x} \\
r(k)=y\left(i_{1}(k)\right) y^{*}\left(i_{2}(k)\right)
\end{gathered}
$$

where $i_{1}(k)$ and $i_{2}(k)$ denote the two chains used in correlator $k$, and

$$
i_{1}=[1,1,1, \ldots, 1,2,2, \ldots, 2, \ldots, M-1]
$$

$$
\begin{aligned}
& i_{2}=[2,3,4, \ldots, M, 3,4, \ldots, M, \ldots, \quad M] \\
& k \in[1,2,3, \ldots \ldots \ldots \ldots \ldots \ldots \ldots \ldots \ldots \ldots, \quad, \quad K]
\end{aligned}
$$

where $K$ is the total number of cross-correlators, equal to

$$
\begin{gathered}
K=\frac{M(M-1)}{2} \\
z=\Re\left(\sum_{k=1}^{K} r(k)\right)=K s^{2}+2 K s x_{r}+K x_{r}^{2}+K x_{m}^{2}+ \\
s \cdot \sum_{k=1}^{K}\left(c_{r}\left(i_{1}(k)\right)+c_{r}\left(i_{2}(k)\right)\right)+\sum_{k=1}^{K} c_{r}\left(i_{1}(k)\right) c_{r}\left(i_{2}(k)\right)+ \\
x_{r} \sum_{k=1}^{K}\left(c_{r}\left(i_{1}(k)\right)+c_{r}\left(i_{2}(k)\right)\right)+\sum_{k=1}^{K} c_{m}\left(i_{1}(k)\right) c_{m}\left(i_{2}(k)\right) \\
+x_{m} \sum_{k=1}^{K}\left(c_{m}\left(i_{1}(k)\right)+c_{m}\left(i_{2}(k)\right)\right)
\end{gathered}
$$

Note that each chain $i$ is used $(M-1)$ times in all the $K$ correlators. Therefore,

$$
\sum_{k=1}^{K}\left(c_{r}\left(i_{1}(k)\right)+c_{r}\left(i_{2}(k)\right)\right)=\sum_{i=1}^{M}(M-1) c_{r}(i)
$$

Similarly,

$$
\sum_{k=1}^{K}\left(c_{m}\left(i_{1}(k)\right)+c_{m}\left(i_{2}(k)\right)\right)=\sum_{i=1}^{M}(M-1) c_{m}(i)
$$

In expression (3.6), $K s^{2}$ is the desired part of the output. All the other terms exist due to the noise. We define $z_{s}$ and $z_{n}$ such that $z_{s}+z_{n}=z$ where $z_{s}$ represents the desired part and $z_{n}$ represents all noise components (all other terms). Including (3.7) and (3.8), this yields

$$
z_{s}=K s^{2}
$$

$$
\begin{gathered}
z_{n}=2 K s x_{r}+K x_{r}^{2}+K x_{m}^{2}+s \sum_{i=1}^{M}(M-1)\left(c_{r}(i)\right)+ \\
\sum_{k=1}^{K} c_{r}\left(i_{1}(k)\right) c_{r}\left(i_{2}(k)\right)+x_{r} \sum_{i=1}^{M}(M-1)\left(c_{r}(i)\right)+ \\
\sum_{k=1}^{K} c_{m}\left(i_{1}(k)\right) c_{m}\left(i_{2}(k)\right)+x_{m} \sum_{i=1}^{M}(M-1)\left(c_{m}(i)\right)
\end{gathered}
$$

The ratio between the power of $z_{s}$ and $z_{n}$ is then defined as $\gamma$. Since we are using FSK as an example and we are looking at the frequency domain, the signal would appear as a discrete delta function. In the designated bin, it would have a constant power.

$$
\gamma=\frac{\left(K \sigma_{s}^{2}\right)^{2}}{\operatorname{Var}\left(z_{n}\right)}
$$


All the terms in $z_{n}$ are uncorrelated so we can calculate the variance of $z_{n}$ by summing their variances.

$$
\begin{aligned}
\operatorname{Var}\left(z_{n}\right)=\frac{M(M-1)^{2}}{2} \sigma_{s}^{2} \sigma_{\mathrm{nc}}^{2} & +\frac{M(M-1)}{4} \sigma_{\mathrm{nc}}^{4} \\
+\frac{M^{2}(M-1)^{2}}{2} \sigma_{s}^{2} \sigma_{\mathrm{nx}}^{2} & +\frac{M(M-1)^{2}}{2} \sigma_{\mathrm{nc}}^{2} \sigma_{\mathrm{nx}}^{2} \\
& +\frac{M^{2}(M-1)^{2}}{4} \sigma_{\mathrm{nx}}^{4}
\end{aligned}
$$

Filling (3.2) into (3.12) gives

$$
\begin{gathered}
\gamma=\frac{M^{2}(M-1)^{2}}{4} \sigma_{s}^{4} /\left(\frac{M(M-1)^{2}}{2} \sigma_{s}^{2}(1-\rho) \sigma_{n}^{2}\right. \\
+\frac{M(M-1)}{4}(1-\rho)^{2} \sigma_{n}^{4}+\frac{M^{2}(M-1)^{2}}{2} \sigma_{s}^{2} \rho \sigma_{n}^{2} \\
\left.+\frac{M(M-1)^{2}}{2} \rho(1-\rho) \sigma_{n}^{4}+\frac{M^{2}(M-1)^{2}}{4} \rho^{2} \sigma_{n}^{4}\right)
\end{gathered}
$$

Using (3.13), we can find an expression for the SNR required at the output of each individual chain to achieve a specific $\gamma$, as a function of $\gamma, M$ and $\rho$.

$$
\begin{gathered}
\text { SNR }=\frac{\sigma_{s}^{2}}{\sigma_{n}^{2}}=\frac{\gamma(1-\rho+M \rho)}{M}+ \\
\sqrt{\frac{\gamma^{2}(1-\rho+M \rho)^{2}}{M^{2}}+\frac{\gamma(1-\rho)^{2}}{M(M-1)}+\frac{2 \gamma \rho(1-\rho)}{M}+} \gamma \\
\sqrt{\gamma \rho^{2}}
\end{gathered}
$$

We can make a few observations from (3.14). First, for $\rho=$ 0 (no noise correlation at all),

$$
\left.\mathrm{SNR}\right|_{\rho=0}=\frac{\gamma}{M}+\sqrt{\frac{\gamma^{2}}{M^{2}}+\frac{\gamma}{M(M-1)}} \propto \frac{1}{M}
$$

SNR is approximately inversely proportional to the number of chains. This can be viewed as a more energy-efficient way to reduce the required SNR compared with long averaging which is inversely proportional to the square root of the number of integrations [4].

Also, for an infinitely high number of chains,

$$
\left.\mathrm{SNR}\right|_{M \rightarrow \infty}=\rho\left(\gamma+\sqrt{\gamma^{2}+\gamma}\right) \propto \rho
$$

Unless $\rho=0$, there is a limit to the reduction in the required SNR. This limit is proportional with $\rho$. Using more chains will not reduce the required SNR below this limit.

It would be more practical to find an equation for SNR (before correlation) as a function of BER, $M$ and $\rho$. Unfortunately, $\gamma$ is not just a function of BER but also depends on $\rho$ and $M$. However, simulations show that if $\rho$ and $M$ are ignored, the error is small (maximum error in $\gamma$ across $\rho$ and $M$ is $13 \%$, which corresponds to a maximum error in SNR of $0.5 \mathrm{~dB}$ as will be shown later), so we calculate $\gamma$ in case of $\rho$ equals $1, \gamma_{1}$, as this also removes the dependency on $M$, and use this value as an approximation. Replacing $\rho=1$ in equation (3.14)

$$
\mathrm{SNR}=\gamma_{1}+\sqrt{\gamma_{1}^{2}+\gamma_{1}} \approx 2 \gamma_{1}+0.5
$$

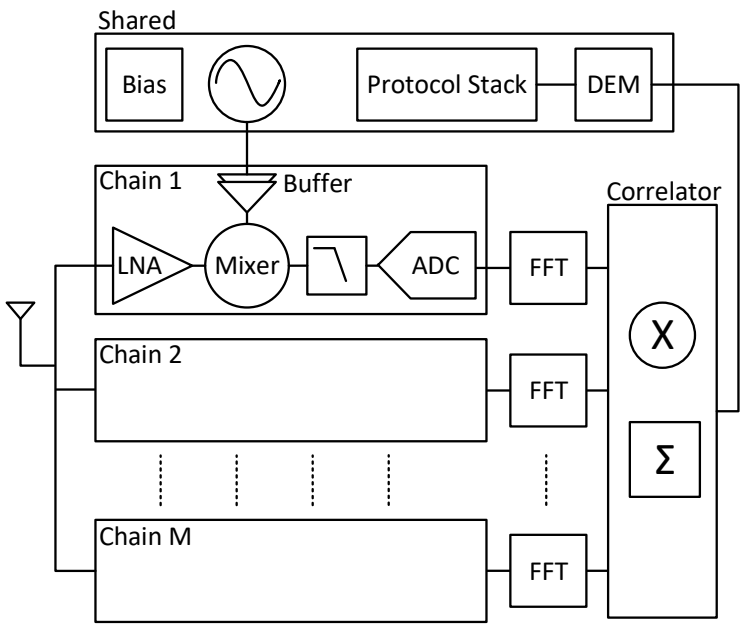

Fig. 4. A model of the proposed receiver used to estimate the power consumption penalty for using multiple chains.

where the last approximation uses a Taylor series expansion. Thus,

$$
\gamma \approx \gamma_{1} \approx \frac{\mathrm{SNR}}{2}-0.25
$$

For non-coherent BFSK modulation, the relation between SNR and BER [5]

$$
\mathrm{BER}=\frac{1}{2} \exp \left(\frac{-\mathrm{SNR}}{2}\right)
$$

So,

$$
\gamma \approx-\ln (2 \mathrm{BER})-0.25
$$

Filling (3.20) in (3.14) produces an equation for SNR as a function of BER, $M$ and $\rho$. We will use this equation to validate the results of this analysis in section IV.

\section{B. Power consumption}

Each additional chain in the receiver increases the overall power consumption. Having an estimate for the additional power consumption would be really useful to decide whether using more chains is worth it or not. For this, we use a simple model for the receiver as shown in Fig. 4.

In this model, the receiver is divided into three different parts. First, a shared part which includes all components that are shared between all the chains such as the LO as discussed in section II, but also biasing circuits, the demodulator, etc. The non-shared part would then need to be copied for the desired number of chains. The same goes for the FFTs as each chain needs its own FFT. Finally, the correlator part consists of multipliers and adders. Since the number of multipliers needed is $M(M-1) / 2$, this part grows faster than the number of chains $M$.

In order to estimate the power consumption for the full receiver for any number of chains, we need an estimate for the power consumption of each of these parts. The shared part is not affected by the number of chains. If some of its components consume more power, e.g. due to driving additional circuits, these contributions should be included in 
the chain part. Fig. 4 shows an LO buffer as a representative example. For the non-shared part, its total power would be the number of chains multiplied by the power consumption of each single chain. For the correlator part, we can estimate to first order that it will grow at the same rate as the number of multipliers.

Using these approximations, we can assume that the power consumption of the complete receiver $P_{\mathrm{Rx}}$ would be:

$$
P_{\mathrm{Rx}}=P_{\text {shared }}+M P_{\text {chain }}+M P_{\mathrm{FFT}}+\frac{M(M-1)}{2} P_{\text {corr }}
$$

where $P_{\text {shared }}$ is the power of the shared part, $P_{\text {chain }}$ is the power of each single chain, $P_{\mathrm{FFT}}$ is the power of each FFT, and $P_{\text {corr }}$ is the power of the correlator for two chains.

To simplify the equations, we define $\alpha$ and $\beta$ as:

$$
\alpha \equiv \frac{P_{\text {chain }}+P_{\text {FFT }}-0.5 P_{\text {corr }}}{P_{\text {shared }}}, \beta \equiv \frac{0.5 P_{\text {corr }}}{P_{\text {shared }}}
$$

which results in

$$
P_{\mathrm{Rx}}=P_{\text {shared }}\left(1+\alpha M+\beta M^{2}\right)
$$

It is apparent from (3.21) that the correlator power may be an issue at a high number of chains. However, as we will show in section IV, it does not become significant in practical scenarios.

\section{Figure of merit}

In order to make sure that the improvement in SNR is worth the higher costs (power), we need to define a FoM that would relate the SNR to the power consumption. In literature of receivers, one popular FoM is the inverse of energy efficiency multiplied by the inverse of sensitivity [6]:

$$
\mathrm{FoM}=\frac{R}{k T \cdot \mathrm{BW} \cdot F \cdot \mathrm{SNR} \cdot P_{\mathrm{Rx}}}
$$

where $k$ is Boltzmann constant, $T$ is the temperature, $\mathrm{BW}$ is the bandwidth, $F$ is the noise factor of the receiver, SNR is the signal-to-noise ratio required at the output of each chain (linear scale) and $R$ is the used data-rate.

Since our main target here is to optimize the number of chains for a specific design, variables such as $R, \mathrm{BW}$ and $F$ are constant and therefore not relevant in our context. For this reason, we define a new FoM $_{s}$ that is a function of only SNR and $P_{\mathrm{Rx}}$

$$
\mathrm{FoM}_{s} \equiv \frac{1}{\mathrm{SNR} \cdot P_{\mathrm{Rx}}}
$$

According to this definition, any design that doubles the power of the receiver should also improve the SNR by $3 \mathrm{~dB}$ or more to be considered efficient. Filling (3.14) and (3.23) into (3.25), we can have an estimate for $\mathrm{FoM}_{s}$ for any number of chains. Fig. 5 shows the optimum number of chains $M_{\text {opt }}$, that maximizes FoM ${ }_{s}$, versus $\rho$ and $\alpha$ for a few different values of $\beta$. It shows that at both low $\rho$ (low noise correlation between chains) and low $\alpha$ (large parts of the receiver can be shared), using more chains will maximize $\mathrm{FoM}_{s}$. This effect is stronger for lower $\beta$ (more power-efficient digital correlators).
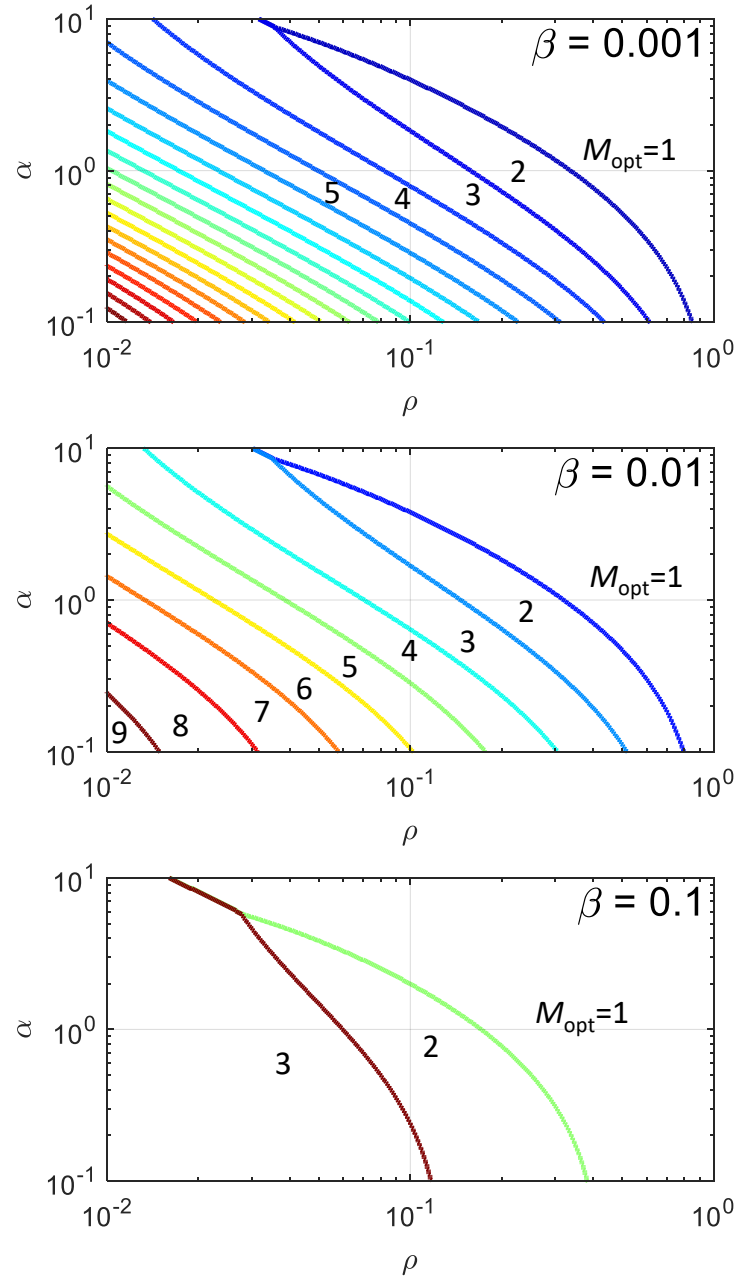

Fig. 5. The optimum number of chains $M_{\mathrm{opt}}$ versus $\rho$ and $\alpha$ at three different values for $\beta$.

\section{Simulation RESUlTS}

In order to verify equation (3.14), Monte Carlo simulations are performed for the demodulation of a BFSK signal using $\mathrm{XC}$ and different number of chains. In every chain, complex Gaussian random noise is created and added to the same signal. The correlation coefficient $\rho$ is varied, and the SNR required to achieve a BER of $10^{-3}$ is determined.

Fig. 6 shows equation (3.14) versus the simulated SNR. Calculations from equation (3.14) fall within $0.5 \mathrm{~dB}$ of the simulated results. The slope of the SNR curves is lower at higher number of chains, i.e. the marginal SNR improvement becomes lower as the number of chains increases. As expected, the higher the correlation between the chains, the less useful adding more chains becomes, since they cannot suppress correlated noise. Fig. 7 shows a simulation of the receiver described in [3] with a different number of chains and zero $\rho$, compared to equation (3.14). Again, the analysis predicts the simulated results well. As shown, the SNR required to get the same BER is lower for a higher number of chains.

We will now show two examples from literature where we 


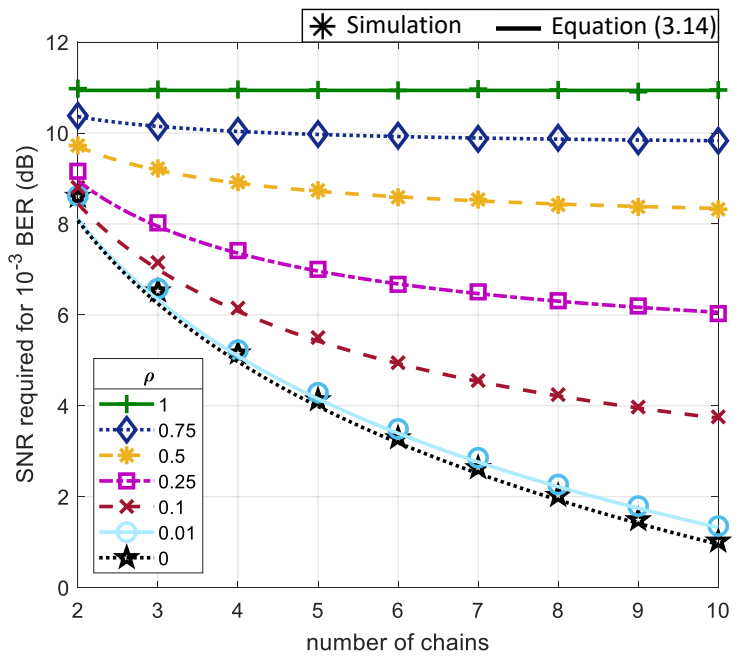

Fig. 6. SNR required for $10^{-3}$ BER in simulation vs. (3.14).

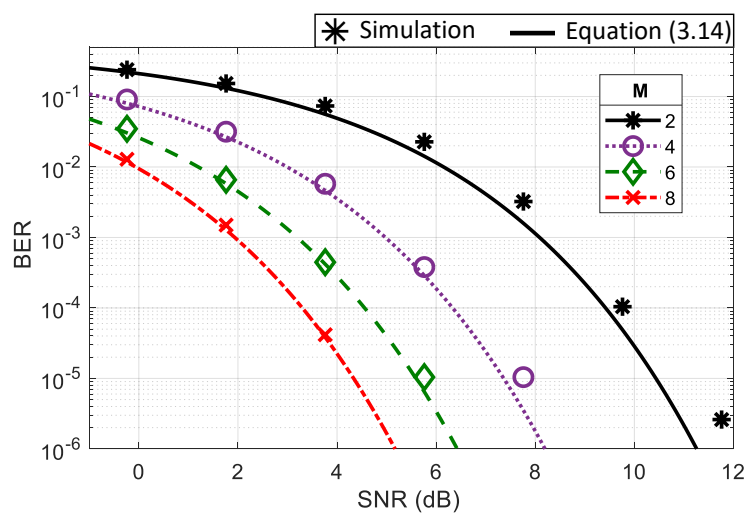

Fig. 7. Simulation results of the receiver described in [3] assuming $\rho=0$ vs. (3.14).

are estimate the effects of increasing the number of chains on their SNR and FoM.

\section{Example 1:}

In [2], XC is used with two chains in an integrated spectrum analyzer ${ }^{1}$. The LNA was designed using two stages: a common-gate (CG) stage (mainly for matching) which consumes $5 \mathrm{~mW}$ and a common source (CS) stage (to boost the gain) which consumes $10.3 \mathrm{~mW}$. The CG power consumption is inversely proportional to the input resistance. Since we are assuming a shared antenna, the same as [2], the power of each CG becomes inversely proportional with the number of chains. Therefore, the total power consumption of all CG stages in all the chains is approximately constant independent of the number of chains. On the other hand, the CS stage has to be replicated for every chain. The LO driver power consumption is $20.4 \mathrm{~mW}$ for $1 \mathrm{GHz}$ frequency. In [2], it was replicated for

\footnotetext{
${ }^{1}$ Note that [2] is not meant to be used as an FSK receiver but we use its parameters to indicate the SNR and FoM improvement in case of using multiple chains.
}
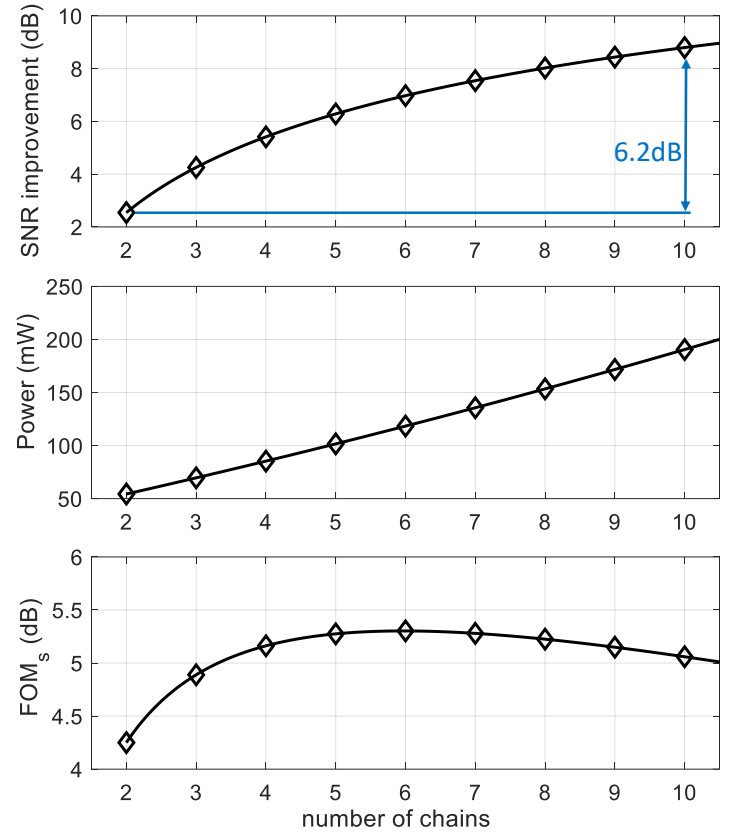

Fig. 8. The SNR improvement, power consumption and FoM for different number of chains in the example in [2]. An improvement of up to $6 \mathrm{~dB}$ can be achieved with virtually no loss in FoM.

each receiver, but it can be shared to save power with an estimated $10 \%$ increase per additional chain for driving the individual mixers. In [2], the FFT needed is 24-bit, 1024point and 20MS/s. Scaling from the results in [7], we estimate the FFT power to be about $2 \mathrm{~mW}$. The correlator used with a 1024-point FFT would only need about $10 \%$ of the FFT power, but to be safe, we estimate a conservative $0.5 \mathrm{~mW}$ for the correlator.

Using these values in (3.22), we estimate $\alpha$ to be $0.55, \beta$ to be 0.01 and $\rho$ is measured as 0.028 [2]. Fig. 8 shows the SNR improvement, power consumption and FoM for different number of chains in this example. It shows that increasing the number of chains from 2 to 10 , would not reduce the FoM while improving the SNR by about $6 \mathrm{~dB}$. In contrast, if integration in time is used, doubling the measurement time improves the SNR by $1.5 \mathrm{~dB}$. To achieve $6 \mathrm{~dB}$ improvement in SNR, 16 integrations would be required which would increase the energy consumption by $12 \mathrm{~dB}$. Hence, if we normalize for the same period, the FoM would be lower by $6 \mathrm{~dB}$.

\section{Example 2:}

In [8], a completely analog FSK receiver is presented using one chain. The components that can be shared such as the LO and the demodulator, amount to about $580 \mu \mathrm{W}$, while the components that need to be replicated, consume about $560 \mu \mathrm{W}$. Since we are using $\mathrm{XC}$ in the digital domain, we still need to add an ADC, FFT and a correlator. Assuming 6 bits (to allow 38dB SQNR so it does not degrade the interference performance) and using 100fJ per conversion step, a $1 \mathrm{MS} / \mathrm{s}$ ADC would consume about $13 \mu \mathrm{W}$. Since this receiver uses 

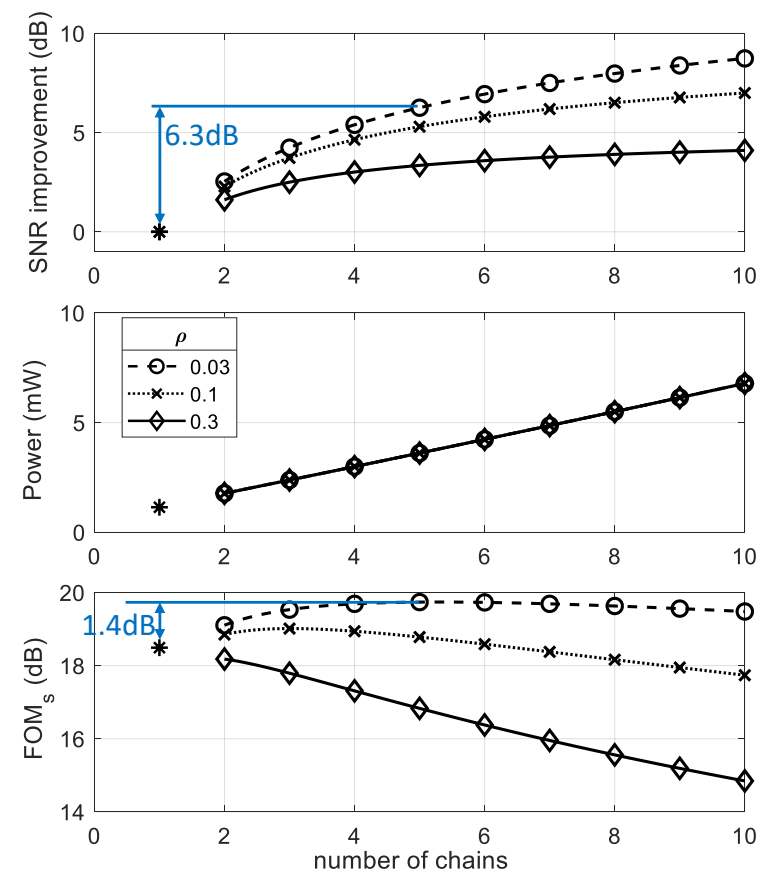

Fig. 9. The SNR improvement, power consumption and FoM for different number of chains assuming different $\rho$ for the example in [8]. Assuming $\rho$ $=0.03$ (best case), FoM $_{s}$ will improve $1.4 \mathrm{~dB}$ and SNR will improve $6.3 \mathrm{~dB}$ using 5 chains instead of 1 .

BFSK, the complete FFT is not useful here, only two multipliers are required to represent the two used frequencies for the demodulation. Using synthesis in TSMC $65 \mathrm{~nm}$ technology, a 6-bit, 8-point and 1MS/s multiplier consumes about $3 \mu \mathrm{W}$ and the correlator about $4 \mu \mathrm{W}$. To be on the safe side, we will double those estimations. This results in $\alpha$ of about 1.02 and $\beta$ around 0.005 .

The noise created in each chain is represented by the NF (in $\mathrm{dB}$ ). Assuming independent noise between the chains, the common noise would be from the antenna and its power is $-\mathrm{NF}(\mathrm{dB})$ compared to the noise at the output of the chain. This results in a $\rho$ which is approximately equal to the inverse of NF (linear scale). In this chip, a NF of $15 \mathrm{~dB}$ was reported which would amount to $\rho \geq 0.03$.

Fig. 9 shows the SNR improvement, power consumption and FoM for different number of chains in this example. It shows that at the best case $(\rho=0.03)$, using 5 chains would improve sensitivity by $6.3 \mathrm{~dB}$ while improving the FoM by $1.4 \mathrm{~dB}$. Even at a pessimistic case $(\rho=0.1)$, the FoM still improves by $0.5 \mathrm{~dB}$ while improving sensitivity by $3.7 \mathrm{~dB}$ using 3 chains.

\section{Discussion:}

In both of these examples, the optimum number of chains is higher than two. We can achieve about 6dB SNR improvement without sacrificing FoM in both cases. This shows the practicality of this approach for many receivers with high NF. Either to improve linearity or to decrease power consumption, a high NF design may be used. Using multiple chains while sharing large portions between them can be useful to improve sensitivity without reducing the FoM of the design.

\section{COnClusions}

$\mathrm{XC}$ is used for many energy detection applications for its noise reduction capabilities. Currently, it is mainly used in applications where data-rate is not a crucial consideration. In this paper, we analyzed the use of multiple chains for noncoherent FSK receivers to improve sensitivity without sacrificing the data-rate. In case of low noise correlation between the chains, the improvement in sensitivity is proportional to the number of chains. Sharing components between the chains is proposed to decrease the power penalty of the added hardware. A figure-of-merit is developed to evaluate the optimum number of chains for different parameters of the receiver design. Two different examples from literature are analyzed and show improvement in sensitivity for multiple chains with similar or even better figure-of-merit. This shows the potential of the proposed solution.

\section{ACKNOWLEDGMENT}

This work is part of the IRUDIT research project (no. 13576), which is financed by the Dutch Research Council (NWO).

\section{REFERENCES}

[1] E. Nilsson and C. Svensson, "Power consumption of integrated low-power receivers," IEEE Journal on Emerging and Selected Topics in Circuits and Systems, vol. 4, no. 3, pp. 273-283, Sep. 2014.

[2] M. S. Oude Alink, E. A. M. Klumperink, A. B. J. Kokkeler, Z. Ru, W. Cheng, and B. Nauta, "Using cross-correlation to mitigate analog/RF impairments for integrated spectrum analyzers," IEEE Transactions on Microwave Theory and Techniques, vol. 61, no. 3, pp. 1327-1337, Mar. 2013.

[3] M. R. M. Mikhael, M. S. Oude Alink, and A. B. J. Kokkeler, "A cross-correlation sub-sampling receiver for low power, low data-rate applications in a low SINR environment," in 2018 IEEE 9th Annual Information Technology, Electronics and Mobile Communication Conference, Vancouver, Canada, Nov. 2018.

[4] M. S. Oude Alink, A. B. J. Kokkeler, E. A. M. Klumperink, G. Smit and B. Nauta, "Lowering the SNR-wall for energy detection using crosscorrelation," IEEE Transactions on Vehicular Technology, vol. 60, no. 8, pp. 3748-3757, 2011.

[5] S. Haykin, Communication systems, 4th ed. Wiley Publishing, 2001.

[6] Y. Liu, A. Ba, J. H. C. van den Heuvel, K. Philips, G. Dolmans, and H. de Groot, "A $1.2 \mathrm{~nJ} / \mathrm{bit} 2.4 \mathrm{GHz}$ receiver with a sliding-IF phase-todigital converter for wireless personal/body area networks," IEEE Journal of Solid-State Circuits, vol. 49, no. 12, pp. 3005-3017, Dec 2014.

[7] T. Yu, C. Yang, D. Cabric, and D. Markovic, "A 7.4-mW 200-MS/s wideband spectrum sensing digital baseband processor for cognitive radios," IEEE Journal of Solid-State Circuits, vol. 47, no. 9, pp. 2235 2245, Sep. 2012.

[8] A. C. Molnar, "Transistors and synapses: robust, low power analog circuits in CMOS radios and the rabbit retina," Ph.D. dissertation, EECS Department, University of California, Berkeley, May 2007. 